\title{
Classification of Melanoma Images Using Modified Teaching Learning Based Artificial Bee Colony
}

\author{
Radhwan Ali Abdulghani Saleh ${ }^{1 *}$, Rustu Akay ${ }^{2}$ \\ ${ }^{1}$ Institute of Natural and Applied Sciences, Erciyes University, Kayseri, Turkey (ORCID: 0000-0001-9945-3672) \\ 2 Department of Mechatronics Engineering, Erciyes University, Kayseri, Turkey (ORCID: 0000-0002-3585-3332)
}

(This publication has been presented orally at HORA congress.)

(First received 1 August 2019 and in final form 24 October 2019)

(DOI: $10.31590 /$ ejosat.637846)

ATIF/REFERENCE: Saleh, R. A. A. \& Akay, R. (2019). Classification of Melanoma Images Using Modified Teaching Learning Based Artificial Bee Colony. European Journal of Science and Technology, (Special Issue), 225-232.

\begin{abstract}
The great improvement in the current technology, particularly in the field of artificial intelligence, has effectively contributed to solving many problems, especially in the medical field. More recently, skin cancer (melanoma) has become one of the most dangerous cancers threatening human life, although it can be treated more frequently at early detection. Unfortunately, only highly-trained specialists can diagnose the disease accurately. Therefore, in this paper we have introduced various software technologies to detect and diagnose skin cancer through images, thus saving lives and reducing the spread of the disease, as well as reducing unnecessary traditional eradication of non-carcinogenic areas. Our method combines image processing techniques (image enhancement, hair removal and segmentation using Otsu's thresholding), feature extraction techniques (Gray Level Co-Occurrence Matrix (GLCM) features and color moments features) and commonly used classification methods, such as Weighted KNN, Cubic SVM, Medium Gaussian SVM, and Multi-Layer Perceptron (MLP) trained by some of the common swarm intelligent techniques like Artificial Bee Colony (ABC), Genetic Algorithm (GA), Particle Swarm Optimization (PSO), Teaching Learning Based Artificial Bee Colony (TLABC), and Modified Teaching Learning Based Artificial Bee Colony (MTLABC) which is the proposed algorithm in this paper. Experimental results for 996 dermoscopy dataset images, show that the classification accuracy and the convergence of the trained Neural Network (NN) using the proposed MTLABC is better than the other evolutionary algorithms used in this study for the same purpose. At the same time, the experimental results show that the classification accuracy of the trained NN using the proposed MTLABC is better than the results of commonly used classification methods.
\end{abstract}

Keywords: TLABC, Modified TLABC, melanoma detection, multi-layer perceptron, classification methods, metaheuristics.

\section{Introduction}

Melanoma is a type of very serious skin cancer. It appears on one part and can spread to other parts of a body. It is linked with skin exposure to ultraviolet (UV) light. It is typically first noticed as a new mole or from the changes happening to an existing one, most commonly in body parts that receive direct sunlight exposure. The mole may be large and appear irregular in shape. Some may have multiple colors or be itchy or bloody. The National Cancer Institute reports that solitary 2 percent of all skin malignancies are melanoma, so it is uncommon. It is additionally perilous. Of a wide range of skin malignant growth, melanoma is the deadliest. In 2017, the National Institute of Health (NIH) gauges that there will be 87,110 new instances of melanoma and 9,730 deaths. The rate of melanoma has expanded in the ongoing years, yet it is not obvious to what degree changes in the environment, in behavior, or early identification is included $[1,2]$.

To reduce the dangers of melanoma, early detection is needed, unfortunately only a highly trained expert can detect melanoma, by using special equipment. This makes the task of detection costly, and time-consuming. Dermoscopy with high-resolution imaging of the skin is one of those methods which reduce the reflection of the skin surface allowing the experts to see deeper to the skin structure. This requires especially highly trained expert clinicians. However, the accuracy of the detection process is based on the trained degree of experts. The number of registered dermatologists in the Middle East \& North Africa is rare, so are highly trained expert clinicians, which make the strongly serious deadliest cancer out of the catch.

\footnotetext{
* Institute of Natural and Applied Sciences, Erciyes University, Kayseri, ORCID: 0000-0001-9945-3672, redhwan9191@gmail.com
} 
In order to solve this problem and make the task of detection easier, a highly trained neural network classification approach is created. The approach has been created to be used as a diagnose tool to help those clinicians who are not highly trained, and it can be used by patients which helps them in the early detection for their moles.

In the literature, there are many researches about skin cancer using different kinds of methods. One of them presents a computeraided algorithm that checks the ABCD features of an image after the segmentation stage. The extracted features are used to classify the image by using the TDS Index [3]. The other research proposes a system for identifying melanoma skin disease with the Otsu thresholding that portions the injury from the whole picture. For more segmentation, the boundary tracing algorithm has been utilized. $\mathrm{ABCD}$ features have been extracted and the Stolz algorithm has been utilized for the classification stage [4]. ABCD features are not the only features which give good results in skin cancer detection application, also GLCM could be used to extract some features such as Entropy, Correlation, Contrast, Homogeneity, Energy, Standard Deviation, Mean, Solidity, Perimeter, Equivdiameter, Area, ConvexArea, Manhattan Distance, Euclidean Distance, Hamming Distance, and Minkowski Distance. GLCM features have been used in many types of research at the stage of extracting features but the classification stage was different as the following: neural networks [5], support vector machine [6], multilayer perceptron classifier [7]. SVM and k-NN classifiers are used for the classification stage with both GLCM features and color features in the feature extraction stage [8].

As described above, the Artificial Neural Network (ANN) is used in the classification stage for skin cancer detection. ANN is a nonlinear and non-parametric model, which is used to solve different types of classification problems. Nonetheless, the convergence of the ordinary training algorithms, such as Back Propagation is slow and not always guaranteed. Therefore, we need efficient optimization strategies to attain faster convergence and higher accuracy rates [9].

Swarm intelligence, which exploits the collective ability of swarm algorithms are popular in solving optimization problems. Also, many studies introduce hybridization between different types of these algorithms, for example, a particle-bee algorithm that integrates the advantages of intelligent behavior of bird swarms and honey bee [10]. Another example is a PS-FW which is a hybrid between fireworks algorithm and PSO particle swarm optimization [11]. Also, hybrid optimization algorithms, which are based on ABC and some other methods, have been developed [12-15]. These hybrid systems have more impact because of their advantages over their individuals.

The power of these hybrid algorithms comes from their individuals that imitating the best features in nature. The success of the hybridization leads the researchers to try different strategies in the global and local search methods to improve the exploitation and exploration of the individual.

In this work, we will first introduce the proposed techniques in the image processing and feature extraction stage. Then we will describe the original TLABC algorithm and its implementation to train the multi-layer perceptron NN. After that, we will formulate the implementation of the modification we proposed to enhance the accuracy of TLABC. Finally, we will perform a comparison between commonly used classification methods, and MLP trained by some of the common swarm intelligent techniques like ABC, GA, PSO, TLABC, and MTLABC.

\section{Proposed techniques in image processing and feature extraction}

\subsection{Pre-processing Step:}

In this step, the sample images are enhanced and improve the restoration of the sample, and any external noise is removed, such as hair. The methods that have been used in this stage are as the following:

- Image contrast enhancement and image rescaling.

- Hair removing using some morphology operations and vision enhancement.

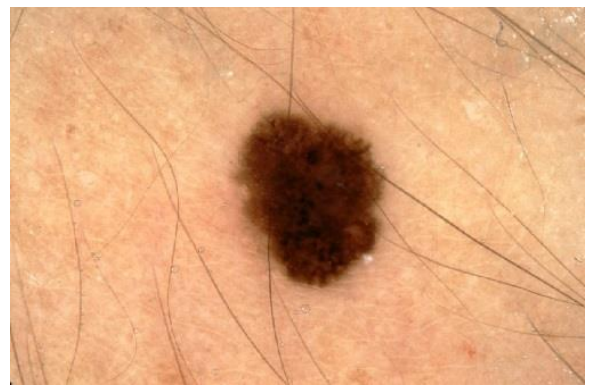

a- Before pre-processing

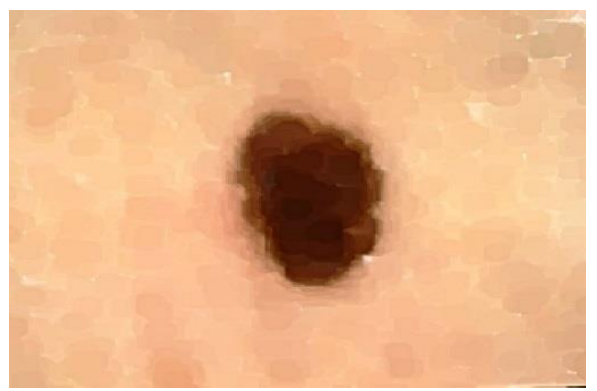

b- After pre-processing

Fig. 1. Sample in the pre-processing step

\subsection{Segmentation Step:}

In this step, a sample taken from the previous step is segmented to separate the lesion area from the background area. In this stage, a morphological operation is used followed by the Otsu's thresholding algorithm. After applying the Otsu's thresholding method and getting the binary mask, the mask then multiplied by the three color channels (Red, Green, and Blue) to extract the region of interest. 


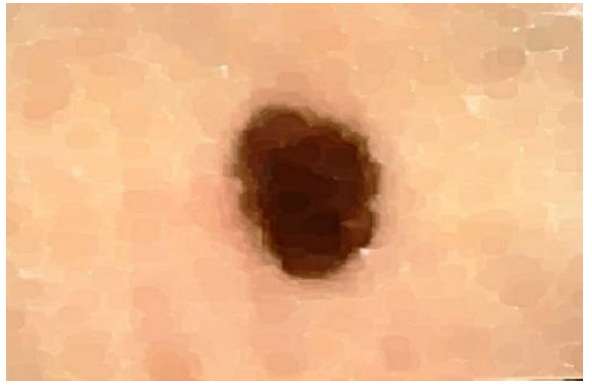

a- Before segmentation

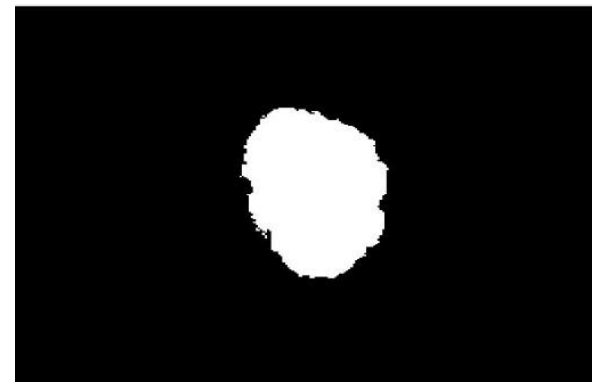

b- using thresholding

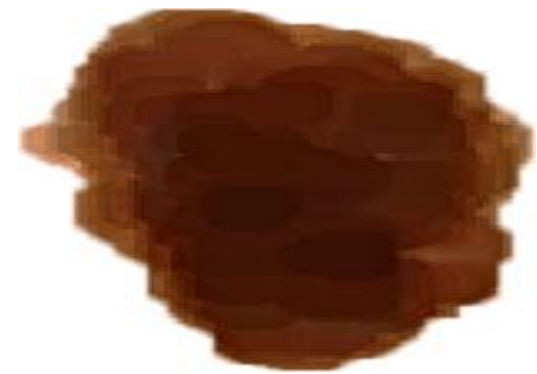

c- result segmentation (lesion Area)

Fig. 2. Sample in the Segmentation step

\subsection{Feature Extraction Step:}

In this step, the lesion sample is taken from the previous step, and its features are extracted and then saved in a vector-matrix. The model that does this process is divided into two parts. The first part for computing the GLCM (Gray Level Co-Occurrence Matrix) and calculating its features, where the derived features from GLCM are four features (Energy, Contrast, Entropy and Homogeneity) from each lesion image The next part is for calculating color features called color moments, this part is dividing image color channels into three channels (red, green, and blue). From each channel, three features can be extracted. So the total number of features extracted from each image are 13 features.

\section{TLABC Algorithm}

TLABC is hybridization between TLBO and ABC algorithm which combines the advantages of both (the exploration of ABC and the exploitation of TLBO). It effectively employs three hybrid search phases as follows [16]:

\subsection{Teaching based employed bee phase}

Here each employed bee uses a hybrid of TLBO and mutation operator of differential evolution to search a new food source, which can develop the variety of search tendencies extraordinarily, and upgrade the searchability of TLABC.

\subsection{Learning-Based Onlooker Bee Phase}

In this stage, an onlooker bee chooses a sustenance source to search out as indicated by the selection probability which is determined to utilize Eq. 6. After that, the onlooker bee finds out new food sources using the TLBO's learning strategy.

\subsection{Generalized Oppositional Scout Bee Phase}

In this stage, if a nourishment source cannot be improved further for a specific period time, it is viewed as depleted and would be relinquished. At that point, an arbitrary candidate solution and the generalized oppositional solution of it are created. The best solution of them is utilized rather than the old depleted nourishment source. The TLABC pseudo-code is shown in Algorithm 1.

\section{Lévy Flight Local Search Algorithm}

The Lévy Flight Local Search is one of the stochastic search algorithms which use a random walk to update its solutions. The step walks are defined as random step lengths, which have a particular probability distribution. These step lengths can be drawn from a Lévy distribution, which is stated in Eq. 6.

$$
\mathrm{L}(\mathrm{s}) \sim|\mathrm{s}|^{-1-\beta}
$$

Where $(0<\beta \leq 2)$ and $s$ is the step length which can be calculated by Eq. 7 .

$$
\mathrm{s}=\frac{\mathrm{u}}{|\mathrm{v}|^{1 / \beta}}
$$

Where $u$ and $v$ are drawn from Gaussian distributions.

$$
\sigma_{\mathrm{u}}=\left\{\frac{\Gamma(1+\beta) \sin (\pi \beta / 2)}{\beta \Gamma[(1+\beta) / 2] 2^{(\beta-1) / 2}}\right\}^{1 / \beta}, \sigma_{\mathrm{v}}=1
$$

The $i$ th individual solution is updated using:

$$
\mathrm{x}_{\mathrm{ij}}^{\prime}(\mathrm{t}+1)=\mathrm{x}_{\mathrm{ij}}(\mathrm{t})+\operatorname{step}_{-} \operatorname{size}(\mathrm{t}) * \mathrm{U}(0,1)
$$

The step sizes of Levy flights are too ornery, that is, they may be used for both exploration and exploitation by changing this step size. The main steps of the LFLS is shown in Algorithm 2 [17]. 
Algorithm 1: Teaching Learning Based Artificial Bee Colony

Step 1: Initialize the population by Eq. 1.

$$
x_{i}^{j}=x_{\text {min }}^{j}+\operatorname{rand}(0,1)\left(x_{\text {max }}^{j}-x_{\text {min }}^{j}\right)
$$

Start by doing the initialization and normalization process of the dataset to be in the range $[-10,10]$.

Select an appropriate number of Input, Output and Hidden neurons for the NN according to the dataset.

Set a suitable maximum number of iterations to train the NN.

\section{REPEAT}

Step 2: Teaching based employed bee phase: To train the NN move the employed bees onto their food sources by using the hybrid of TLBO and mutation operator of differential evolution in Eq. 2.

$$
u_{i, d}=\left\{\begin{array}{c}
x_{i, d}^{\text {old }}+\operatorname{rand}_{2}\left(x_{\text {teacher }, d}-T_{F} \cdot x_{\text {mean,d }}\right), \\
x_{r 1, d}+F .\left(x_{r 2, d}-x_{r 3, d}\right), \text { if } \text { otherwise }_{1}<0.5
\end{array}\right.
$$

Pass the obtained food sources to train the NN.

Calculate the NN's mean square error and compute its classification accuracy.

IF The new food sources are better than the older

Replace the new instead of the old solutions

\section{ELSE}

Increase the failure

END IF

Step 3: Learning based onlooker bee phase: Determine the nectar amounts of food sources by Eq. 3.

$$
\mathrm{P}_{\mathrm{i}}=\frac{\mathrm{fit}\left(x_{i}\right)}{\sum_{\mathrm{i}=1}^{\mathrm{SN}} \operatorname{fit}\left(x_{i}\right)}
$$

Using the roulette method, some onlooker bees will be selected to move onto the food sources according to the selection probability of Eq. 3 .

The selected onlooker bees will move onto the food sources using the TLBO's learning strategy in Eq. 4.

$$
u_{s}=\left\{\begin{array}{l}
x_{s}+\text { rand } .\left(x_{s}-x_{j}\right), \text { if } f\left(x_{s}\right) \leq f\left(x_{j}\right) \\
x_{s}+\text { rand } .\left(x_{j}-x_{s}\right), \text { if } f\left(x_{j}\right)>f\left(x_{s}\right)
\end{array}\right.
$$

Pass the obtained food sources to train the NN.

Calculate the NN's mean square error and compute its classification accuracy.

IF The new food sources are better than the older

Replace the newer instead of the older solutions

\section{ELSE}

Increase the failure

\section{END IF}

Step 4: Generalized oppositional scout bee phase: An arbitrary candidate solution and the generalized oppositional solution of it are created using Eq. 1 and Eq. 5 respectively.

$$
\mathrm{x}_{\mathrm{i}, \mathrm{j}}^{\mathrm{GO}}=\mathrm{k} \cdot\left(\mathrm{a}_{j}+\mathrm{b}_{j}\right)-x_{i, j}
$$

The best solution of them is utilized rather than the old depleted nourishment source.

UNTIL requirements are met.

Algorithm 2: Lévy Flight Local Search Strategy

Insert the optimization function $\operatorname{Min} \mathrm{f}(\mathrm{x})$ and $\beta$;

An individual $x_{i}$ will be selected randomly then initialize $\mathrm{t}=1$ and $\sigma_{\mathrm{v}}=1$;

Compute $\sigma_{\mathrm{u}}$ using Eq. 8

WHILE $(\mathrm{t}<\varepsilon)$ do

Use Eq. 7 to compute step size;

Use Eq. 9 to generate a new solution $\mathrm{x}_{\mathrm{i}}^{\prime}$ then calculate $\mathrm{f}\left(\mathrm{x}_{\mathrm{i}}^{\prime}\right)$;

IF $\mathrm{f}\left(\mathrm{x}_{\mathrm{i}}^{\prime}\right)<\mathrm{f}\left(\mathrm{x}_{\mathrm{i}}\right)$

$\mathrm{x}_{\mathrm{i}}=\mathrm{x}_{\mathrm{i}}^{\prime}$;

END IF

$\mathrm{t}=\mathrm{t}+1$;

END WHILE

Return the $x_{i}$ 


\section{The proposed modification of TLABC Algorithm (MTLABC)}

As mentioned above, TLABC has a good balance between exploration and exploitation but actually this modification will significantly enhance its performance. The proposed modification of TLABC Algorithm is a kind of low-level integrative hybridization between TLABC and Levy Flight algorithm. The good exploration of ABC attracts a lot of researchers to hybrid it with many other swarm algorithms as we mentioned above. Some of them keep both the equations of searching and the framework of $A B C$ too, but the others keep just the framework of ABC. In TLABC hybridization just the framework was keeping, so, in this paper, we modified the search operaters defined by Eq. 10 and Eq. 11. The pseudo-code for this modification is shown in Algorithm 3.

Algorithm 3: Modified Teaching Learning Based Artificial Bee Colony

Step 1: Initialize the population by Eq. 1.

Start by doing the initialization and normalization process of the dataset to be in the range [-10, 10].

Select an appropriate number of Input, Output and Hidden neurons for the NN according to the dataset.

Set a suitable maximum number of iterations to train the NN.

\section{REPEAT}

Step 2: Modified employed bee phase: To train the NN moves the employed bees onto their food sources by using the hybrid of ABC's employed bees strategy and mutation operator of differential evolution in Eq. 10.

$$
u_{i, d}=\left\{\begin{array}{l}
x_{i, j}+\operatorname{rand}(0,1)\left(x_{i, j}-x_{j, k}\right), \text { if rand } 1<0.5 \\
x_{r 1, d}+F .\left(x_{r 2, d}-x_{r 3, d}\right), \text { otherwise }
\end{array}\right.
$$

Pass the obtained food sources (weights) to train the NN.

Calculate the NN's mean square error and compute its classification accuracy.

IF The new food sources are better than the older

Replace the new instead of the old solution

\section{ELSE}

Increase the failure

\section{END IF}

Step 3: Learning based onlooker bee phase: Determine the nectar amounts of food sources by Eq. 3.

Using the roulette method some onlooker bees will be selected to move onto the food sources according to the selection probability of Eq. 3 .

The selected onlooker bees will move onto the food sources using the hybrid of TLBO's learning strategy and the LF local searching strategy in Eq. 11.

$$
u_{i, j}=\left\{\begin{array}{cl}
\mathrm{x}_{\mathrm{s}}+\text { rand } .\left(\mathrm{x}_{\mathrm{s}}-\mathrm{x}_{\mathrm{j}}\right), \& \text { if } \mathrm{f}\left(\mathrm{x}_{\mathrm{s}}\right) \leq \mathrm{f}\left(\mathrm{x}_{\mathrm{j}}\right) & \text { if } \text { rand }_{1}<0.5 \\
\mathrm{x}_{\mathrm{s}}+\operatorname{rand} .\left(\mathrm{x}_{\mathrm{j}}-\mathrm{x}_{\mathrm{s}}\right), \& \text { if } \mathrm{f}\left(\mathrm{x}_{\mathrm{j}}\right)>\mathrm{f}\left(\mathrm{x}_{\mathrm{s}}\right), & \text { otherwise } \\
\mathrm{x}_{\mathrm{ij}}(\mathrm{t})+\text { step } \mathrm{p}_{\text {size }(\mathrm{t})} * \mathrm{U}(0,1), &
\end{array}\right.
$$

Pass the obtained food sources to train the NN.

Calculate the NN's mean square error and compute its classification accuracy.

IF The new food sources are better than the older

Replace the newer instead of the older solutions

ELSE

END IF

Increase the failure

Step 4: Generalized oppositional scout bee phase: An arbitrary candidate solution and the generalized oppositional solution of it are created using Eq. 1 and Eq. 5 respectively.

The best solution of them is utilized rather than the old depleted nourishment source.

UNTIL requirements are met.

\section{Computational Results}

The inputs to the NN are the thirteen features of the lesion sample. The outputs are the class labels that classify the lesion to one of two classes (Melanoma or Normal). A number of hidden nodes is 15 with one hidden layer. The dataset is divided into $85 \%$ training and $15 \%$ testing. The tenfold cross validation is performed and the average of ten folds is recorded as the classification accuracy. We compared MTLABC with other models like TLABC, ABC, GA, and PSO for the same purpose of classification and the results of all of them are summarized in terms of convergence curves of mean square error in Fig. 3. Table 1 illustrates the accuracy of NN classification after training using these algorithms for 1000 evaluations.

The following configuration was considered in the experiments:

- The initialization within the search space was uniform random.

- $\quad$ Limit $=100$.

- $\quad$ The Number of food sources $\mathrm{SN}=50$.

- The range search was inside the interval $[-10,10]$.

- The configurations for other algorithms are the same as the proposed algorithm. 
All experiments were implemented 30 times with different random seeds, and all classification algorithms in this paper are coded in MATLAB R2018b. Table 1 shows the classification accuracy of the trained MLP using TLABC, ABC, GA, PSO and the proposed MTLABC on the testing dataset.

The proposed MTLABC enhances the performance of the training of the NN to overcome the local minimum of the NN since it enhances the possibility of exploiting and exploration of TLABC because of the diversity resulted from the used searching equations in both employed and onlooker bees' phases. This is illustrated by the continuously decreasing in Mean Square Error (MSE) values during the training as shown in Fig. 3. Figure 3 shows the result of a sample of the 30 experiments such that the results of the remaining 29 experiments show the same trend.

Table 1. Classification Rates of MLP trained using TLABC, ABC, GA, PSO and the proposed MTLABC on the testing dataset

\begin{tabular}{|c|c|c|c|c|c|}
\hline & \multicolumn{5}{|c|}{ Classification Accuracy \% } \\
\hline $\begin{array}{ll} & \text { Training } \\
\text { No Of. } & \text { Algorithm } \\
\text { Experiment }\end{array}$ & MTLABC & TLABC & $\mathrm{ABC}$ & GA & PSO \\
\hline 1 & 72.28916 & 77.71084 & 74.09639 & 71.68675 & 69.27711 \\
\hline 2 & 77.10843 & 76.50602 & 73.49398 & 70.48193 & 75.30120 \\
\hline 3 & 80.12048 & 75.30120 & 78.31325 & 74.09639 & 71.08434 \\
\hline 4 & 77.71084 & 71.08434 & 75.30120 & 71.68675 & 71.68675 \\
\hline 5 & 75.90361 & 73.49398 & 74.09639 & 71.68675 & 68.67470 \\
\hline 6 & 73.49398 & 75.30120 & 72.28916 & 73.49398 & 71.08434 \\
\hline 7 & 74.69880 & 74.09639 & 68.07229 & 72.28916 & 70.48193 \\
\hline 8 & 76.50602 & 74.69880 & 70.48193 & 70.48193 & 68.07229 \\
\hline 9 & 73.49398 & 76.50602 & 71.68675 & 68.67470 & 68.67470 \\
\hline 10 & 73.49398 & 74.69880 & 77.10843 & 74.69880 & 68.07229 \\
\hline 11 & 74.69880 & 75.30120 & 72.28916 & 73.49398 & 72.28916 \\
\hline 12 & 74.09639 & 75.30120 & 73.49398 & 74.09639 & 66.26506 \\
\hline 13 & 75.90361 & 73.49398 & 75.90361 & 77.10843 & 67.46988 \\
\hline 14 & 75.90361 & 74.09639 & 74.09639 & 67.46988 & 74.09639 \\
\hline 15 & 72.89157 & 75.90361 & 73.49398 & 71.68675 & 66.26506 \\
\hline 16 & 77.10843 & 74.09639 & 74.69880 & 70.48193 & 68.67470 \\
\hline 17 & 76.50602 & 72.28916 & 71.68675 & 72.89157 & 71.08434 \\
\hline 18 & 74.69880 & 73.49398 & 72.89157 & 69.27711 & 68.07229 \\
\hline 19 & 75.30120 & 75.30120 & 76.50602 & 63.25301 & 71.08434 \\
\hline 20 & 76.50602 & 74.09639 & 73.49398 & 71.68675 & 73.49398 \\
\hline 21 & 71.68675 & 77.10843 & 70.48193 & 72.89157 & 70.48193 \\
\hline 22 & 77.10843 & 68.07229 & 75.90361 & 71.08434 & 68.07229 \\
\hline 23 & 72.28916 & 72.89157 & 73.49398 & 74.09639 & 74.69880 \\
\hline 24 & 74.09639 & 75.30120 & 71.08434 & 69.27711 & 72.89157 \\
\hline 25 & 74.69880 & 74.69880 & 74.09639 & 75.30120 & 70.48193 \\
\hline 26 & 76.50602 & 74.69880 & 72.28916 & 69.87952 & 66.26506 \\
\hline 27 & 74.69880 & 75.30120 & 75.30120 & 69.87952 & 66.26506 \\
\hline 28 & 75.30120 & 74.69880 & 75.30120 & 72.28916 & 72.28916 \\
\hline 29 & 74.69880 & 74.69880 & 72.28916 & 75.30120 & 68.67470 \\
\hline 30 & 74.09639 & 74.09639 & 71.08434 & 73.49398 & 65.66265 \\
\hline Mean & 75.12050 & 74.47790 & 73.49400 & 71.80720 & 69.89960 \\
\hline Standard deviation & 1.83910 & 1.82090 & 2.20920 & 2.72820 & 2.68700 \\
\hline Max & 80.12050 & 77.71080 & 78.31330 & 77.10840 & 75.30120 \\
\hline Min & 71.68670 & 68.07230 & 68.07230 & 63.25300 & 65.66270 \\
\hline
\end{tabular}

According to the results in Fig. 3, which is a sample of convergence curves of the training process, the proposed MTLABC has the best convergence speed over all other training algorithms used in this paper. Also, the accuracy of the NN classification increases as the number of evaluations increases even if it exceeds a large number of evaluations, unlike some of the other algorithms. The reason for this increase is due to the advantages of using different kinds of the searching process in each phase of algorithm phases. 


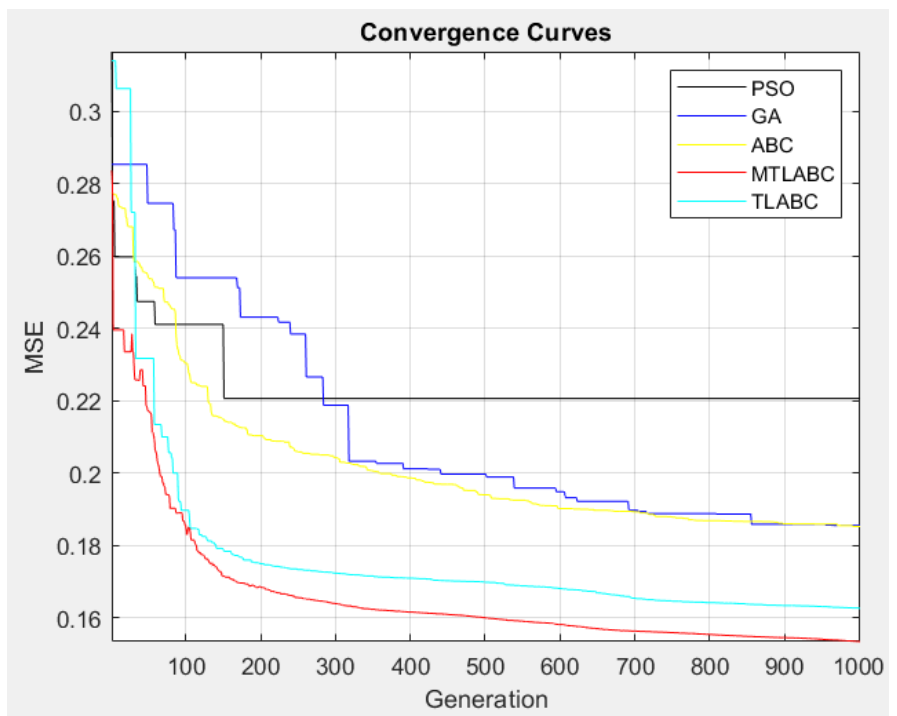

Fig. 3. Sample of convergence curves of mean square error while training process of MLP using TLABC, ABC, GA, PSO, and the proposed MTLABC

The same dataset also used to train commonly used classification methods for the same purpose. Table 2 illustrates the comparison between the classification accuracy of the best three methods and the average of the classification accuracies of the proposed MTLABC which is clarified in Table 1. As it is clear from Table 2, the average of the classification accuracies of the proposed algorithm is better than all commonly used classification methods.

Table 2. Comparison Between The Proposed MTLABC and Commonly Used Classification Methods In Terms Of Classification Rates

\begin{tabular}{|c|c|c|c|}
\hline \multicolumn{3}{|c|}{ Classification Accuracy \% } \\
\hline MTLABC & Weighted KNN [19] & Cubic SVM [20] & Medium Gaussian SVM [20] \\
\hline 75.1205 & 74.1 & 73.6 & 73.4 \\
\hline
\end{tabular}

\section{Conclusions and Recommendations}

In this paper, we concentrated on the modification of Teaching Learning Based Artificial Bee Colony algorithm, which is more efficient than the original TLABC on training multi-layer perceptron NN for the dermoscopy dataset of 996 lesion images. This modification uses the advantages of LF algorithm to improve the exploitation of TLABC. The experiments are performed on the dataset [18] after applying image processing techniques (image enhancement, hair removal and segmentation using Otsu's thresholding), feature extraction techniques (GLCM features and color moments features). The results of this work show that the accuracy of the NN classifier which is trained by using the proposed MTLABC is more accurate than TLABC, ABC, GA, PSO algorithms. The results also show that the accuracy of the NN classifier trained by using the proposed MTLABC is more accurate than commonly used classification methods on the same dataset. We recommend to use this proposed algorithm to train NNs on other applications, especially those whom their dataset more complicated.

\section{References}

[1] Kelly, J. W., Yeatman, J. M., Regalia, C., Mason, G., \& Henham, A. P. (1997). A high incidence of melanoma found in patients with multiple dysplastic naevi by photographic surveillance. Medical journal of Australia, 167(4), 191-194.

[2] LIVESCIENCE. https://www.livescience.com/34783-uv-rays-increase-melanoma-skin-cancer-risk.html. [Accessed 17 2019].

[3] Jaiswar, S., Kadri, M., \& Gatty, V. (2015). Skin Cancer Detection Using Digital Image Processing. International Journal of Scientific Engineering and Research, 3(6), 138-140.

[4] Gopinathan, S., \& Rani, S. N. A. (2016). The melanoma skin cancer detection and feature extraction through image processing techniques. Orthopedics, 5(11).

[5] Al-Amin, M., Alam, M. B., \& Mia, M. R. (2015). Detection of Cancerous and Non-cancerous Skin by using GLCM Matrix and Neural Network Classifier. International Journal of Computer Applications, 132(8), 44.

[6] Ansari, U. B., \& Sarode, T. (2017). Skin cancer detection using image processing. Int Res J Eng Technol, 4(4), $2875-2881$.

[7] Sheha, M. A., Mabrouk, M. S., \& Sharawy, A. (2012). Automatic detection of melanoma skin cancer using texture analysis. International Journal of Computer Applications, 42(20), 22-26.

[8] Sumithra, R., Suhil, M., \& Guru, D. S. (2015). Segmentation and classification of skin lesions for disease diagnosis. Procedia Computer Science, 45, 76-85. 
[9] Rohra, J. G., Perumal, B., Narayanan, S. J., Thakur, P., \& Bhatt, R. B. (2017). User localization in an indoor environment using fuzzy hybrid of particle swarm optimization \& gravitational search algorithm with neural networks. In Proceedings of Sixth International Conference on Soft Computing for Problem Solving (pp. 286-295). Springer, Singapore.

[10]Lien, L. C., \& Cheng, M. Y. (2012). A hybrid swarm intelligence based particle-bee algorithm for construction site layout optimization. Expert Systems with Applications, 39(10), 9642-9650.

[11] Chen, S., Liu, Y., Wei, L., \& Guan, B. (2018). PS-FW: a hybrid algorithm based on particle swarm and fireworks for global optimization. Computational intelligence and neuroscience, 2018.

[12] Tsai, P. W., Pan, J. S., Shi, P., \& Liao, B. Y. (2011). A new framework for optimization based-on hybrid swarm intelligence. In Handbook of Swarm Intelligence (pp. 421-449). Springer, Berlin, Heidelberg.

[13] Jadon, S. S., Tiwari, R., Sharma, H., \& Bansal, J. C. (2017). Hybrid artificial bee colony algorithm with differential evolution. Applied Soft Computing, 58, 11-24.

[14]Li, X., Peng, Z., Du, B., Guo, J., Xu, W., \& Zhuang, K. (2017). Hybrid artificial bee colony algorithm with a rescheduling strategy for solving flexible job shop scheduling problems. Computers \& Industrial Engineering, 113, 10-26.

[15] Chengli, F. A. N., Qiang, F. U., Guangzheng, L. O. N. G., \& Qinghua, X. I. N. G. (2018). Hybrid artificial bee colony algorithm with variable neighborhood search and memory mechanism. Journal of Systems Engineering and Electronics, $29(2), 405-414$.

[16] Chen, X., \& Xu, B. (2018, June). Teaching-learning-based artificial bee colony. In International Conference on Swarm Intelligence (pp. 166-178). Springer, Cham.

[17] Sharma, H., Bansal, J. C., \& Arya, K. V. (2013). Opposition based lévy flight artificial bee colony. Memetic Computing, 5(3), 213227.

[18] International Skin Imaging Collaboration.” Dataset images”. [Online]. Available: http://www.dermoscopy.org. [accessed 172019$].$

[19] Cost, S., \& Salzberg, S. (1993). A weighted nearest neighbor algorithm for learning with symbolic features. Machine learning, $10(1), 57-78$.

[20] Cristianini, N., \& Shawe-Taylor, J. (2000). An introduction to support vector machines and other kernel-based learning methods. Cambridge university press. 